\title{
Descripción de las Corrientes en un Tubo Elevador a través de la Distribución Gamma
}

\section{M.G. Félix-Flores ${ }^{1^{*}}$, José R. Hernández-Barajas ${ }^{2}$ y R. Vázquez-Román ${ }^{3}$}

(1) Universidad Autónoma de Zacatecas, Unidad Académica de Ciencias Químicas,

Ciudad Universitaria Siglo XXI, Edificio 6, Carr. Zacatecas-Guadalajara km 6, Ejido "La Escondida", 98160 Zacatecas, Zac.-México (e-mail: g_felixflores@hotmail.com)

(2) Universidad Juárez Autónoma de Tabasco, División de Ciencias Biológicas, Carr. VillahermosaCárdenas km 0.5, 86039 Villahermosa, Tab.-México (e-mail: roberto.hernandez@dacbiol.ujat.mx)

(3) Instituto Tecnológico de Celaya, Departamento de Ingeniería Química, Av. Tecnológico s/n, 38010 Celaya, Gto.-México (e-mail: richart@iqcelaya.itc.mx).

*autor a quien debe ser dirigida la correspondencia

\begin{abstract}
Resumen
En este trabajo se describen las corrientes de entrada y salida del tubo elevador de las unidades de desintegración catalítica, utilizando la termodinámica continua. Este tipo de corrientes contiene un número grande de componentes y la termodinámica continua, a través de una función de distribución, permite definir composiciones por grupos de componentes. Se determinan los parámetros de la distribución gamma de las corrientes para varios casos de operación industrial. Los resultados indican que la termodinámica continua predice bien la distribución de los componentes de las corrientes del tubo elevador. Se concluye que la metodología se podría aplicar en una refinería para evaluar la composición de los productos de desintegración catalítica en un intervalo amplio de masas moleculares.
\end{abstract}

\section{Description of the Riser Streams through the Distribution Gamma Function}

\begin{abstract}
The objective of the work is to describe the feed and product streams of the riser of fluid catalytic cracking units using continuous thermodynamic. These streams contain a large number of components and continuous thermodynamic can estimate composition by groups of components, using a distribution function. The parameters of the gamma distribution of the feed and product streams for several industrial cases were determined. The results indicate that continuous thermodynamic can well predict the distribution of the components of the streams in the riser. It is concluded that the proposed methodology can be applied in a refinery to evaluate the composition of the products of catalytic disintegration in a broad interval of molecular mass.
\end{abstract}

Keywords: fluid catalytic cracking, riser, gamma distribution, continuous thermodynamics 


\section{INTRODUCCIÓN}

La función del proceso de desintegración catalítica en lecho fluidizado (FCC del inglés fluid catalytic cracking) es convertir fracciones pesadas del petróleo de elevado peso molecular a productos de menor peso molecular y de mayor valor comercial como la gasolina de alto octano y olefinas (Avidan y Shinnar, 1990; Sadeghbeigi, 2000). El proceso consiste de varias etapas: precalentamiento de la alimentación, vaporización de la alimentación, reacción de desintegración, la separación de los productos del catalizador y regeneración del catalizador, Fig. 1. La alimentación se inyecta en el fondo del tubo elevador y se vaporiza al ponerse en contacto con el catalizador regenerado para llevar a cabo las reacciones endotérmicas en flujo ascendente. La alimentación típica del proceso es un gasóleo de elevado peso molecular que tiene un punto de ebullición promedio entre 600 y $800 \mathrm{~K}$. Después de la reacción, la corriente que sale del tubo elevador se transfiere a un sistema de ciclones para separar los productos de desintegración del catalizador. Los productos son enviados a una torre de destilación y el catalizador con aquellos hidrocarburos que aún se encuentran sobre la superficie catalítica son tratados con vapor de agua en un agotador. El coque que se produce durante las reacciones de desintegración se deposita sobre la superficie del catalizador disminuyendo su actividad. Una combustión del coque permite restaurar la actividad catalítica.

Las mezclas en las corrientes del tubo elevador contienen una gran cantidad de compuestos de peso molecular diverso. La termodinámica continua ha sido una alternativa para describir el comportamiento de mezclas continuas o compleja (Cotterman et al., 1985; Riazi, 2005). Una mezcla continua contiene un número grande de componentes donde resulta conveniente describir la composición de la mezcla a través de una función de distribución de alguna propiedad. Baer et al. (1997), acoplan la termodinámica continua y los modelos de los coeficientes de actividad para describir el equilibrio líquido-vapor. Sin embargo, su aplicación es válida a bajas presiones pero extienden su aplicación a otras condiciones usando ecuaciones de estado cúbicas y grupos de contribución. Vakili-Nezhaad et al. (2001), utilizan la ecuación de Antoine para evaluar la presión de vapor de mezclas continuas ideales y sus resultados concuerdan con datos experimentales. Además, ellos introducen tanto la versión continua de la ley de Raoult como la del coeficiente de actividad de la ecuación de UNIFAC para determinar el equilibrio líquido-vapor de mezclas continuas no ideales. Fang y Lei (2003), también evalúan la presión de vapor y el equilibrio líquido-vapor de estas mezclas; sin embargo, utilizan la distribución gaussiana en función de la temperatura de ebullición para representar su composición.

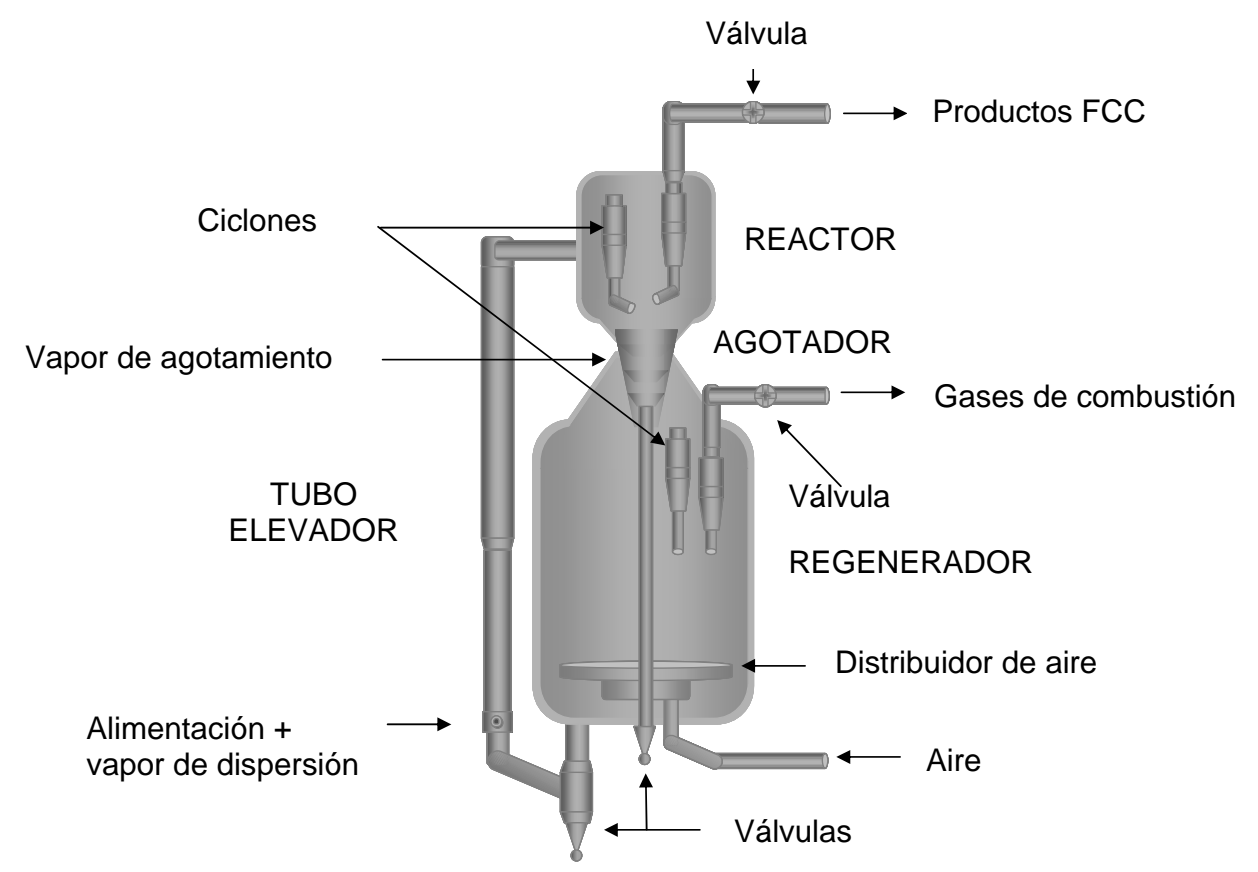

Fig. 1: Unidad de desintegración catalítica. 
Arias-Zugasti y Rosner (2003), utilizan tanto el método del Galerkin como el método de colocación ortogonal para describir la vaporización y la combustión de la gasolina y el diesel. La gasolina y el diesel están constituidos por un número grande de componentes por lo cual son en realidad mezclas continuas. Abdel-Qader y Hallett (2005), analizan el efecto de mezclado en la evaporación de mezclas continuas atomizadas y establecen que tal efecto es insignificativo en este tipo de mezclas. Behrenbruch y Dedigama (2006), utilizan la distribución gamma con dos parámetros para caracterizar la curva TBP de 24 mezclas de hidrocarburos. Además, correlacionan los parámetros de la función de distribución de las mezclas para determinar la densidad relativa. Asimismo, relacionan estos parámetros con la fracción de destilado en un diagrama triangular. Lage (2007), propone un método para caracterizar una mezcla continua, el cual está basado en la conservación de los métodos estadísticos. Los resultados sugieren que este método supera al método de la cuadratura gaussiana en la caracterización de este tipo de mezclas. Browarzik (2007), a partir de la termodinámica continua, propone un modelo para describir el equilibrio de fases para sistemas binarios en el cual uno de sus componentes es asociado. El modelo analiza 15 sistemas del tipo no polar (A) más un componente (B) asociado en donde el componente asociado puede ser metanol, etanol, anilina o agua. El modelo se basa en la energía de Gibbs en exceso y calcula entalpías de exceso, el equilibrio líquido-vapor y/o el equilibrio líquido-liquido. La cinética continua se ha aplicado para estudiar las reacciones de desintegración térmica, de polimerización y la degradación de sistemas polidispersos (Mccoy, 1996; Browarzik y Kehlen, 1997). La desintegración térmica de las mezclas complejas requiere que se defina una expresión del coeficiente estequiométrico que indique la distribución de los productos y otra de la expresión de velocidad en función del tamaño de las moléculas que se desintegran. Las constantes de velocidad de las reacciones de polimerización y de la degradación de sistemas polidispersos se asumen independientes de la masa molar de las especies que reaccionan y, además, eligen una distribución inicial de la expresión de la velocidad continua. La función de distribución utilizada es la de Schulz-Flory y sus parámetros dependen del tiempo de reacción.

El objetivo de este trabajo es desarrollar, a partir de información conocida de la refinería, una metodología que sea capaz de analizar la posibilidad de aplicar la termodinámica continua en la descripción de las corrientes alimentadas y generadas en el tubo elevador de la unidad de desintegración catalítica. La termodinámica continua para describir una mezcla requiere la distribución de alguna propiedad medible la cual, según el contenido de las especies, será ajustada a la forma de la distribución (Riazi, 2005). Así, los datos provenientes de una refinería son ajustados a la función de distribución gamma y la forma de ésta depende del contenido de las corrientes del tubo elevador.

\section{METODOLOGÍA}

La función de distribución gamma, $F(P M)$, ha usado al peso molecular, $P M$, como variable distribuida (Cotterman et al., 1985; Riazi, 2005):

$F(P M)=\frac{(P M-\gamma)^{\alpha-1} \exp \left[-\frac{P M_{7+}-\gamma}{\beta}\right]}{\beta^{\alpha} \Gamma(\alpha)}$

donde $\Gamma$ es la función gamma y $\alpha, \beta$, y $\gamma$ son parámetros de la función de distribución gamma. $\alpha$ proporciona la forma de la distribución, $\gamma$ es el valor mínimo de la variable distribuida (en este caso el peso molecular inicial de la mezcla compleja), y $\beta$ se calcula en función del peso molecular promedio $\mathrm{PM}_{7_{+}}$de la mezcla:

$\beta=\frac{P M_{7_{+}}-\gamma}{\alpha}$

La integral de la función de distribución gamma desde $\gamma$ hasta $P M_{i}$, representa la función de probabilidad acumulada y es equivalente a la fracción peso 0 mol acumulada de un pseudocomponente.

Información Tecnológica Vol. - 19 No3 - 2008 
$P(P M)=\int_{\gamma}^{P M_{i}} F(P M) d P M$

donde $P\left(P M_{i}\right)$ representa el área bajo la curva de $F(P M)$ desde $\gamma$ hasta $P M_{i}$. Cuando $P M_{i}$ tiende al infinito el valor de $P\left(P M_{i}\right)$ se aproxima a la unidad. La fracción mol o peso $Z_{i}$ de un pseudocomponente i que se encuentra en el intervalo $P M_{i-1}$ hasta $P M_{i}$, es evaluada por la integral de $F(P M)$ en ese intervalo, Fig. 2. Un pseudocomponente es un agrupamiento de varias especies químicas que se encuentran dentro de un intervalo de peso molecular o temperatura de ebullición.

$Z_{i}=\int_{P M_{i-1}}^{P M_{i}} F(P M) d P M=P\left(P M_{i}\right)-P\left(P M_{i-1}\right)$

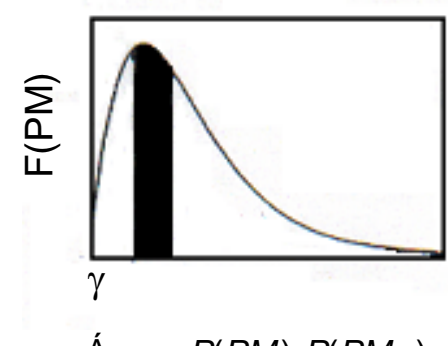

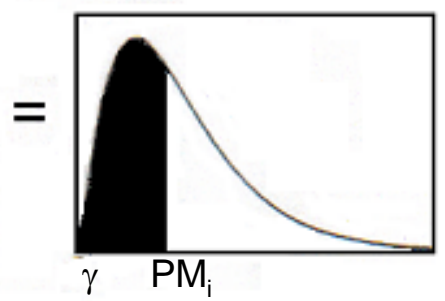

Área $=P\left(P M_{i}\right)$

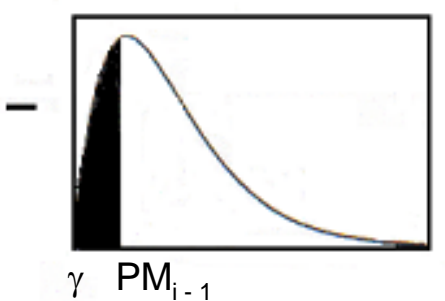

Área $=P M\left(P M_{i-1}\right)$

Fig. 2: Representación gráfica de la relación entre la función de probabilidad, $P(P M)$, y la fracción mol o peso acumulada de una mezcla continua.

El peso molecular promedio $P M_{i}$ de este pseudocomponente está dado por la siguiente expresión:

$P M_{i}=\frac{\int_{P M_{i-1}}^{P M_{i}} P M F(P M) d P M}{Z_{i}}$

y el peso molecular promedio de la mezcla continua se expresa:

$P M_{7+}=\int_{\gamma}^{*} P M F(P M) d P M=\alpha \beta+\gamma=Z_{i} P M_{i}$

En la literatura, Félix-Flores (2001) propone un algoritmo de termodinámica continua para determinar los parámetros de la función de distribución gamma de una mezcla continua. El algoritmo consiste en: a) Dividir "arbitrariamente" la mezcla en $n$ pseudocomponentes y proporcionar el peso molecular y la fracción mol o masa de cada uno de ellos, el peso molecular mínimo ( $\gamma$ ), y un valor inicial del parámetro $\alpha$; b) Calcular la fracción mol de cada pseudocomponente, con la Ecuación 5, entre los límites inferior y superior:

$L I=\frac{P M_{\exp _{i}}+P M_{\exp _{i-1}}}{2}$

$L S=\frac{P M_{\exp _{i}}+P M_{\exp _{i+1}}}{2}$

c) Calcular la suma de los errores de la fracción mol, $\Sigma\left(Z_{i}^{\text {exp }}-Z_{i}^{\text {cal }}\right)^{2}$ y d) Proponer un nuevo valor para el parámetro $\alpha$ para regresar al inciso a hasta que se encuentre el valor óptimo de este parámetro.

El parámetro $\alpha$ de la función de distribución gamma de las corrientes de alimentación y de los productos del tubo elevador es calculado a partir de información que se encuentra comúnmente disponible en una refinería, como las curvas de destilación ASTM-D1160 y ASTM-D86 (Gray, 1994; 
Sadeghbeigi, 2000; Riazi, 2005). Las curvas de destilación proporcionan información sobre la calidad y composición de una fracción del petróleo. La norma ASTM-D1160 describe aquellas fracciones de petróleo que contienen compuestos de alto peso molecular como la alimentación al tubo elevador. La norma ASTM-D86 describe aquellas fracciones de petróleo que contienen compuestos de bajo peso molecular como los productos del tubo elevador. Para determinar el parámetro $\alpha$ de los productos se tomaron en cuenta diferentes esquemas de agrupamiento: 6, 9, 13, 21, 29 y 30 pseudocomponentes. El esquema de 6 pseudocomponentes está conformado por fracciones identificables a escala industrial tales como aceite-cíclico pesado, ACP, $\left(T_{b}: 648-810 \mathrm{~K}\right)$; aceite-cíclico ligero, $\mathrm{ACL},\left(T_{b}\right.$ : 550- $648 \mathrm{~K})$; gasolina pesada, GP, ( $T_{b}$ : 475 - $\left.550 \mathrm{~K}\right)$; gasolina ligera, $\mathrm{GL},\left(T_{b}: 309-475 \mathrm{~K}\right)$; butanos - butilenos, $\mathrm{C}_{4},\left(T_{b}: 231-309 \mathrm{~K}\right)$ y gases ligeros $\left(\mathrm{C}_{1}-\mathrm{C}_{3}, \mathrm{H}_{2}\right.$ y $\left.\mathrm{H}_{2} \mathrm{~S}, T_{b}<231 \mathrm{~K}\right)$. Los esquemas de agrupamiento propuestos toman en cuenta la subdivisión de los pseudocomponentes identificables a partir de las curvas de destilación de los productos de refinería. Así, a partir de las propiedades de la alimentación y del catalizador y de las condiciones de operación del tubo elevador es posible realizar una regresión lineal múltiple para evaluar el parámetro $\alpha$ de la función de distribución gamma de los productos. La mezcla reactiva es subdividida en pseudocomponentes identificables para evaluar los parámetros de la función de distribución gamma en función de la posición longitudinal en el tubo elevador (z). Posteriormente, se emplea una función racional tipo Padé para correlacionar $\alpha$ en función de $z$. El parámetro $\alpha$ es entonces descrito por la siguiente expresión:

$$
\alpha=\frac{a_{0}+a_{1} z+a_{2} z^{2}+a_{3} z^{3}+a_{4} z^{4}}{1+a_{5} z+a_{6}^{2}+a_{7}^{3}} \quad a_{i}=b_{0, i}+b_{1, i} \rho_{\text {relativa }}^{\text {Alim }}+b_{2, i} T_{b}^{\text {Alim }}
$$

Nótese que los parámetros de la función tipo Padé son, a su vez, una función de la densidad relativa, $\rho_{\text {relativa, }}^{\text {Alim }}$ y la temperatura de ebullición de la alimentación, $T_{b}^{\text {Alim }}$, del proceso de desintegración catalítica. La $\rho_{\text {relativa }}^{\text {Alim }}$ se obtiene de los datos de planta y $T_{b}^{\text {Alim }}$ es el MeABP (por sus siglas en inglés mean average boiling point), y se evalúan con una correlación empírica que utiliza los puntos de la curva ASTM-D1160 (Sadeghbeigi, 2000; Riazi, 2005).

\section{RESULTADOS Y DISCUSIÓN}

Para llevar a cabo este trabajo, se empleó una base de datos de 26 casos de operación de una unidad industrial con capacidad promedio de procesamiento de 25,000 barriles diarios y orientada a la producción de gasolinas ligeras. Estos datos se resumen en la Tabla 1.

Tabla 1: Datos de operación de una unidad de desintegración catalítica.

\begin{tabular}{|c|c|c|c|c|c|}
\hline \multirow{2}{*}{ Alimentación } & \multicolumn{2}{|c|}{ Valor } & \multirow{2}{*}{ Regenerador } & \multicolumn{2}{|c|}{ Valor } \\
\hline & mínimo & máximo & & mínimo & máximo \\
\hline Densidad relativa $20 / 4^{\circ} \mathrm{C}$ & 0.8801 & 0.9232 & $\begin{array}{l}\text { Temperatura del catalizador } \\
\text { regenerado }(\mathrm{K})\end{array}$ & 912 & 972 \\
\hline $\begin{array}{l}\text { ASTM-D1160, K } \\
\text { 10\% vol }\end{array}$ & 570 & 637 & $\begin{array}{l}\text { Coque sobre el catalizador } \\
\text { regenerado } \%(\chi)\end{array}$ & 0.05 & 0.95 \\
\hline $30 \%$ & 619 & 677 & Presión (bar) & 2.5 & 2.5 \\
\hline $50 \%$ & 660 & 717 & MAT en equilibrio (\%) & 65 & 74 \\
\hline $70 \%$ & 694 & 750 & Rendimientos (\% masa) & & \\
\hline $90 \%$ & 728 & 796 & ACP & 2.3 & 11.9 \\
\hline Entrada al tubo elevador & & & ACL & 1.6 & 17.3 \\
\hline Flujo de gasóleo (kg/s) & 26 & 43 & Gasolina pesada & 6.4 & 13.5 \\
\hline Temperatura del gasóleo (K) & 432 & 488 & Gasolina ligera & 44.9 & 51.7 \\
\hline Flujo de catalizador (kg/s) & 273 & 468 & $\mathrm{C}_{4 ' s}$ & 7.6 & 11.3 \\
\hline Flujo de vapor de agua $(\mathrm{kg} / \mathrm{s})$ & 1.15 & 1.15 & $\mathrm{C}_{3^{\prime} \mathrm{s}}$ & 7.0 & 13.8 \\
\hline Temperatura del vapor (K) & 573 & 573 & Coque & 3.8 & 6.0 \\
\hline & & & Temperatura final de productos (K) & 787 & 808 \\
\hline
\end{tabular}

La Fig. 3 muestra las composiciones experimentales y calculadas con el algoritmo de Félix-Flores (2001). Los resultados permiten inferir que el algoritmo predice favorablemente la distribución de las 
especies contenidas en la alimentación del proceso de desintegración catalítica. La Fig. 4 muestra la descripción de una corriente de alimentación a través de la función de distribución gamma. Se emplean regresiones lineales múltiples debido a que se pretende obtener un modelo empírico lo más simple posible. Debido a su naturaleza lineal, las regresiones son calculadas a partir del método de mínimos cuadrados. Así, las regresiones lineales múltiples que se obtuvieron para cada esquema de agrupamiento son:

\section{3 pseudocomponentes}

$$
\begin{aligned}
& \alpha^{\text {Prod }}=7.1442 \alpha^{\text {Alimen }}-0.01107 \beta^{\text {Alimen }}+0.24465 \gamma^{\text {Alimen }}-0.00503 T_{\text {mez }}- \\
& 0.00506(C / A)+0.01918 \chi+0.30114 \text { MAT }+0.34819 \rho_{\text {relativa }}^{\text {Alimen }}-10.23492
\end{aligned}
$$

\section{1 pseudocomponentes}

$$
\begin{aligned}
& \alpha^{\text {Prod }}=5.6265 \alpha^{\text {Alim }}-0.00755 \beta^{\text {Alim }}+0.23953 \gamma^{\text {Alim }}-0.00402 T_{\text {mez }}-0.00545(C / A)+ \\
& 0.01802 \chi+0.25923 M A T+0.30240 \rho_{\text {relativa }}^{\text {Alim }}-7.6736
\end{aligned}
$$

donde $\alpha^{\text {Alim }}, \beta^{\text {Alim }}$ y $\gamma^{\text {Alim }}$ y $\rho_{\text {relativa }}^{\text {Alim }}$ son los parámetros de la distribución gamma y la densidad relativa de la alimentación; $T_{\text {mez }}$, temperatura de mezclado; C/A, $\mathrm{kg}$ de catalizador/kg de la alimentación; $\chi, \mathrm{kg}$ de coque/ $\mathrm{kg}$ de catalizador y MAT (del inglés, microactivity test) es una medida de la actividad y selectividad entre la combinación alimentación-catalizador. La $T_{m e z}$ se obtiene a través de un balance de energía en la base del tubo elevador que incluye la alimentación, el vapor de dispersión, coque y el catalizador regenerado (Arandes et al., 2000). Con los esquemas de agrupamiento de 13 y 21 pseudocomponentes se obtuvieron los modelos empíricos con mayor capacidad de predicción en los productos. El modelo empírico con el esquema de 13 pseudocomponentes tienen mayor capacidad de predicción en el rendimiento de los gases y el modelo empírico con el esquema de 21 pseudocomponentes tiene mayor capacidad de predicción en la GP y en el ACP+ACL+GP. La Fig. 5 muestra los resultados de la regresión lineal múltiple de los modelos empíricos con los esquemas de agrupamiento para obtener el parámetro $\alpha$ de los productos. El ajuste de los datos con los modelos 6 , 9, 29 y 30 pseudocomponentes no existió una mejora significativa con respecto a los modelos de 13 y 21 pseudocomponentes. Los parámetros de la función racional de Padé que permite la estimación del parámetro $\alpha$ en función de la posición axial del tubo elevador se muestran en la Tabla 2. La subrutina GREG (Stewart et al., 1992) se utilizó para estimar los parámetros de la función de Padé (Burden y Faires, 2002).

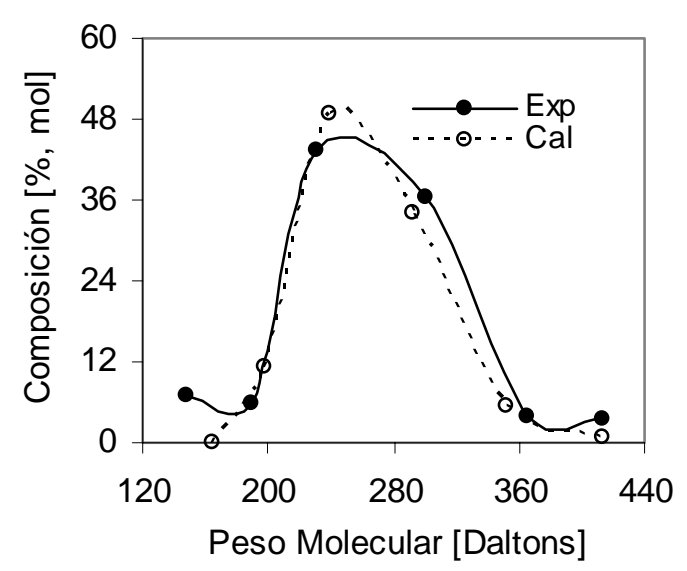

Fig. 3: Comparación de composiciones calculadas con el algoritmo de optimización y las obtenidas a partir de la curva ASTM-D1160 para una alimentación del proceso.

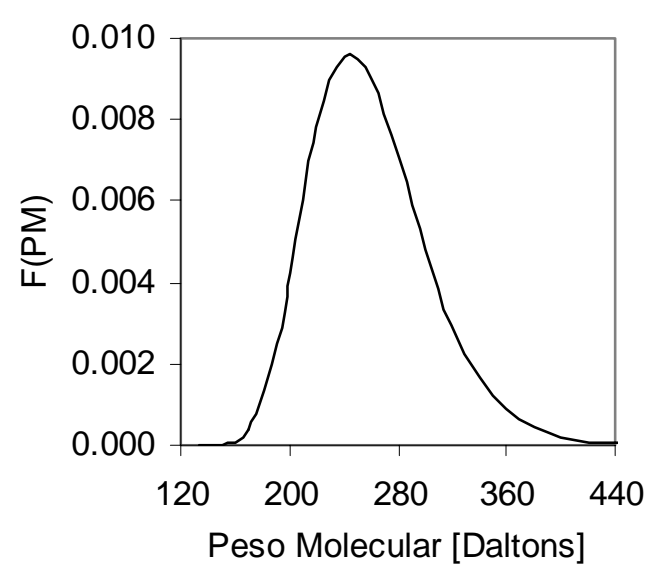

Fig. 4: Descripción de la alimentación del proceso FCC a través de la función de distribución gamma, con $\alpha=8.301, \beta=15.167$ y $\gamma=133$. 


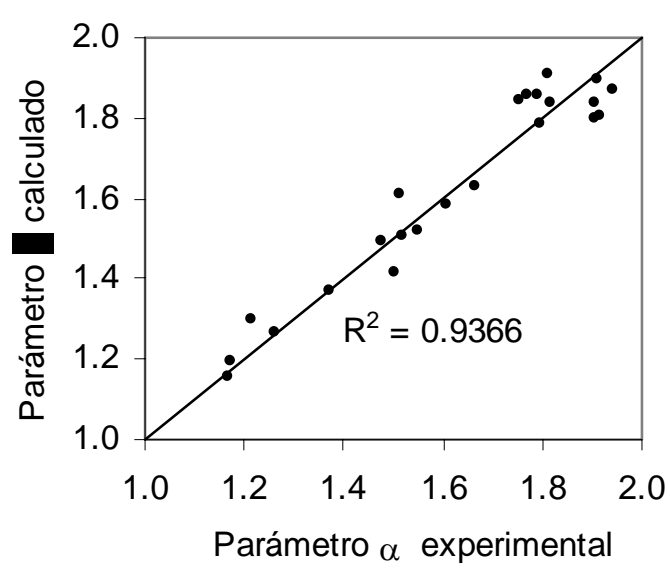

a) 13 pseudocomponentes

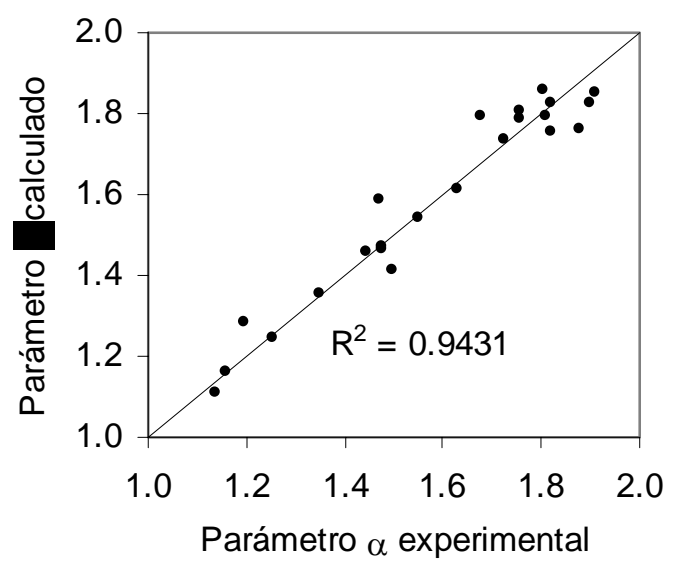

b) 21 pseudocomponentes

Fig. 5: Capacidad de predicción de las correlaciones del parámetro $\alpha$ de la función de distribución gamma de los productos FCC.

Tabla 2: Parámetros de la función racional de Padé

\begin{tabular}{|c|c|c|c|c|c|c|c|}
\hline$b_{00}=$ & 65.71244 & $b_{06}=$ & -24304.94403 & $b_{14}=$ & 22.61762 & $b_{22}=$ & 46.42417 \\
\hline$b_{01}=$ & 1305.61599 & $b_{07}=$ & -1076.74390 & $b_{15=}$ & 571.81182 & $b_{23}=$ & 19.61387 \\
\hline$b_{02}=$ & -27554.04049 & $b_{10}=$ & 129.75999 & $b_{16}=$ & 49.93645 & $b_{24}=$ & -0.05073 \\
\hline$b_{03}=$ & -2404.95993 & $b_{11}=$ & -2752.01398 & $b_{17}=$ & -7326.08355 & $b_{25}=$ & -2.29003 \\
\hline$b_{04}=$ & 12.52582 & $b_{12}=$ & -2853.16993 & $b_{20}=$ & -0.26696 & $b_{26}=$ & 49.93645 \\
\hline$b_{05}=$ & 966.06273 & $b_{13}=$ & -11667.06183 & $b_{21}=$ & 1.72088 & $b_{27}=$ & 11.64351 \\
\hline
\end{tabular}

La Fig. 6 muestra la distribución gamma de una mezcla reactiva a lo largo del tubo elevador. Además, se observa que la forma de la distribución gamma de la alimentación cambia rápidamente debido a que la mayor parte de la conversión del gasóleo a especies ligeras ocurre en los primeros metros. Por ello, la distribución gamma de la mezcla reactiva se desplaza hacia los pesos moleculares más bajos conforme avanza la reacción. Al conocer el parámetro $\alpha$ con el algoritmo de optimización a lo largo del tubo elevador, se procedió a correlacionar 26 casos de operación industrial para obtener los parámetros de la relación tipo Padé.

La Fig. 7 indica que la relación tipo Padé tiene la capacidad de predecir el parámetro $\alpha$ de la función de distribución gamma. Además, muestra que el parámetro $\alpha$ aumenta paulatinamente al inicio del tubo elevador para posteriormente mantenerse casi constante. Sin embargo, el peso molecular de la mezcla reactiva (mezcla continua) cambia con respecto a la longitud del tubo elevador y, por lo tanto, cambia el parámetro $\beta$ de acuerdo a la Ecuación 2. Como el $\mathrm{H}_{2}$ se hace presente durante la reacción de desintegración, se le asigna al parámetro $\gamma$ el valor de cero en toda la longitud del tubo elevador. En resumen, al cambiar los valores de los parámetros cambia también la escala y forma de la función de distribución gamma, ver Fig. 6.

A partir de información disponible de refinería, Félix-Flores (2006) obtiene los factores pre-exponenciales de las constantes cinéticas de un modelo cinético de desintegración catalítica de 6 pseudocomponentes: $\mathrm{ACP}, \mathrm{ACL}, \mathrm{GP}, \mathrm{GL}, \mathrm{C}_{4^{\prime} \text { s }}$ y $\mathrm{C}_{3^{\prime} \text { s }}$ y los rendimientos calculados de los pseudocomponentes se aproximan a los rendimientos de planta. Además, el perfil longitudinal de estos rendimientos coinciden con los reportados en la literatura (Dewachtere et al., 1999; Han y Chung, 2001). La Fig. 8 muestra los rendimientos de los productos de desintegración identificables con respecto a la longitud del tubo elevador de un caso de operación. Además, indica que en los primeros 10 metros del tubo elevador se llevan a cabo casi la totalidad de las reacciones de desintegración; por tal motivo, podemos concluir nuevamente que las distribuciones en los primeros metros cambian rápidamente y en los últimos el cambio es poco significativo, como lo muestra la Fig. 6. 


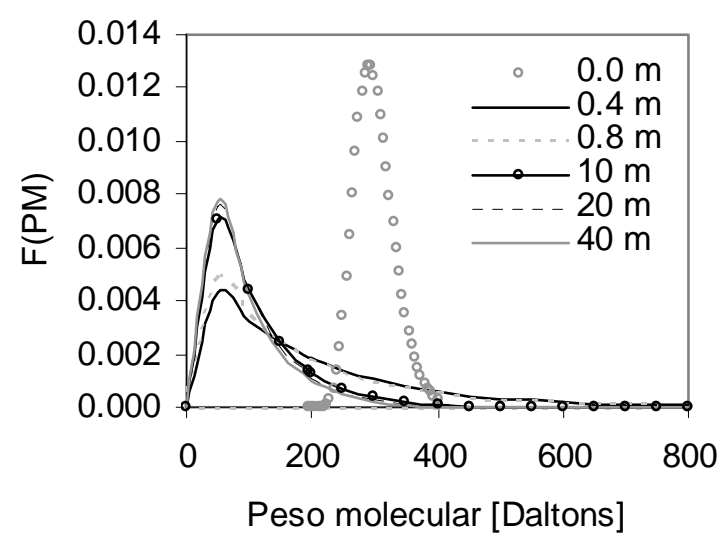

Fig. 6: Perfil longitudinal de la distribución gamma en el tubo elevador.

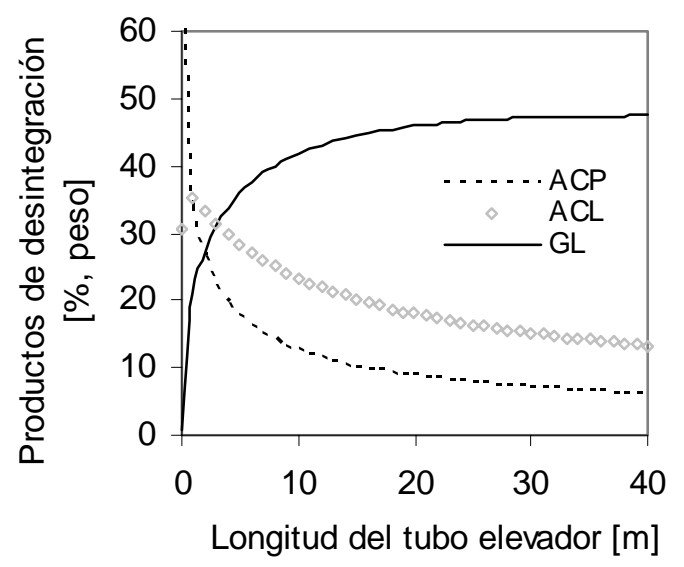

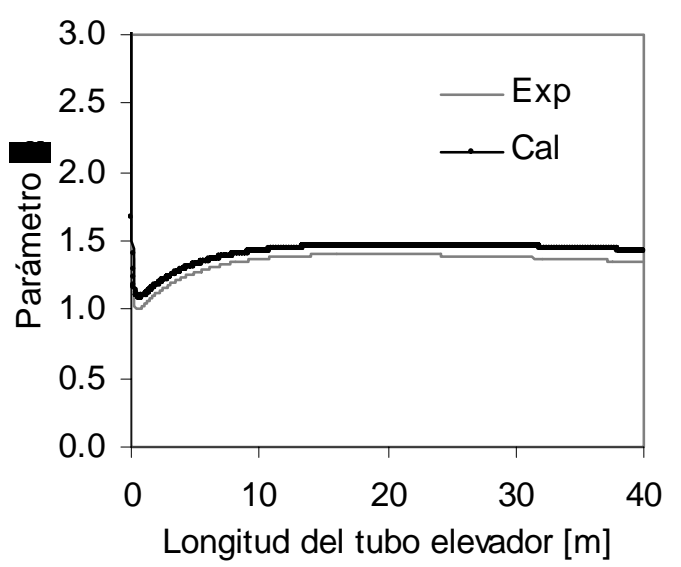

Fig. 7: Comparación entre el parámetro $\alpha$ calculado con el algoritmo de optimización y con la regresión tipo Padé.

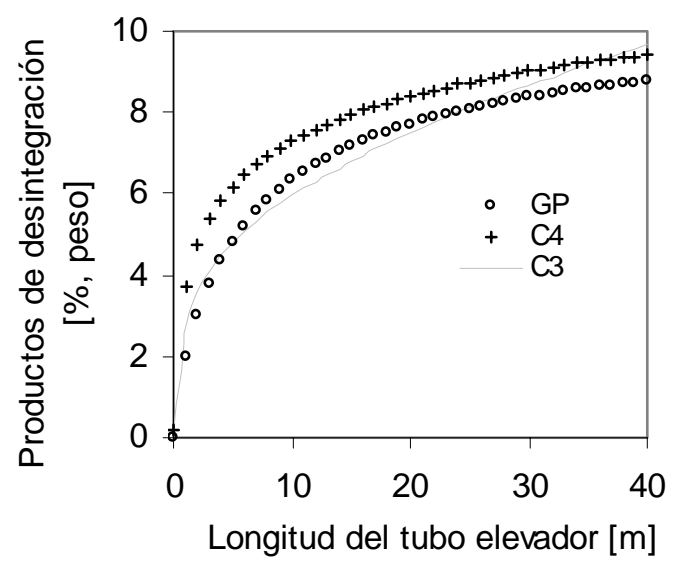

Fig. 8: Perfiles axiales de los productos de desintegración: a) ACP, ACL y GL; b) GP, $C_{4}, C_{3}$

\section{CONCLUSIONES}

El algoritmo de optimización a partir del concepto de termodinámica continua, al proporcionarle información industrial típica, es capaz de predecir favorablemente la distribución de las especies químicas de las corrientes de entrada y salida del tubo elevador del proceso FCC.

El factor de correlación $R^{2}$ del esquema de 21 pseudocomponentes es mayor al de los otros esquemas, lo cual indica que el esquema de 21 pseudocomponentes supera a los otros esquemas para correlacionar las variables de operación con el parámetro $\alpha$ de los productos y en predecir los rendimientos de los productos del proceso FCC.

Finalmente, se sugiere que la relación tipo Padé es adecuada para predecir el parámetro $\alpha$ de la mezcla reactiva a lo largo del tubo elevador. Una vez conocidos los parámetros de la función de distribución gamma $(\alpha, \beta, \gamma)$ en función de la longitud podremos describir la composición de intervalos de peso molecular de la mezcla reactiva en cada posición longitudinal del tubo elevador y establecer estrategias de operación para maximizar productos en función de las demandas del mercado.

\section{AGRADECIMIENTOS}

Los autores agradecen el apoyo de CONACYT, CONCYTEG y a la Coordinación de Investigación y Posgrado de la Universidad Autónoma de Zacatecas. 


\section{NOMENCLATURA}

$\begin{array}{ll}C / A & \text { Catalizador/alimentación, } \mathrm{kg} / \mathrm{kg} \\ F & \text { Función de distribución gamma } \\ M A T & \text { Actividad catalítica del catalizador en equilibrio } \\ P M & \text { Peso molecular } \\ P M_{i} & \text { Peso molecular del pseudocomponente } i \\ P\left(P M_{i}\right) & \text { Probabilidad del pseudocomponente } i \\ P M_{7+} & \text { Peso molecular promedio de la mezcla continua } \\ T_{b} & \text { Temperatura de ebullición, } \mathrm{K} \\ T_{\text {mez }} & \text { Temperatura de mezclado, } \mathrm{K} \\ Z & \text { Longitud en el tubo elevador, } \mathrm{m} \\ Z_{i} & \text { Fracción masa o mol del pseudocomponente } i \\ Z_{\text {ical }} & \text { Fracción masa o mol calculada del pseudocomponente } i \\ Z_{\text {iexp }} & \text { Fracción masa o mol experimental del pseudocomponente } i \\ \alpha, \beta, \gamma & \text { Parámetro de la función de distribución gamma } \\ \chi & \text { Relación coque/catalizador, kg/kg } \\ \Gamma & \text { Función gamma } \\ \rho_{\text {relativa }} & \text { Densidad relativa de la alimentación del proceso de desintegración catalítica }\end{array}$

\section{REFERENCIAS}

Abdel-Qader Z. y W.L.H. Hallett; The Role of Liquid Mixing in Evaporation of Complex Multicomponent mixtures: Modelling Using Continuous Thermodynamics, Chemical Engineering Science: 60(6) 16291640 (2005).

Arandes J.M., N.J. Azkoiti, J. Bilbao y H. I. de Lasa; Modelling FCC Units under Steady and Unsteady Status Conditions, Can. J. Chem. Eng.: 78, 111-123 (2000).

Arias Zugasti M. y D.E. Rosner; Multicomponent Fuel Droplet Vaporization and Combustion using Espectral Theory for a Continuous Mixture, Combustion and Flame: 135(3), 271-284 (2003).

Avidan A.A. y R. Shinnar; Development of Catalytic Cracking Technology. A lesson in Chemical Reactor Design, Industrial Engineering Chemistry Research: 29(6), 931-942 (1990).

Behrenbruch P. y T. Dedigama; Classification and Characterization of Crude Oils Based on Distillation Properties, Journal of Petroleum Science and Engineering: 57(1-2), 166-180 (2007)

Baer U., D. Browarzik y H. Kehlen; Thermodynamics of Semicontinuous Mixtures Using Equations of State with Group Gontributions, Fluid Phase Equilibria: 124(1-2), 29-44 (1997).

Burden R.L. y J.D. Faires; Análisis Numérico. Séptima edición, 520-524, Thomson Learning, México, (2002).

Browarzik D. y H. Kehlen; Continuous Kinetics of Reversible Reactions in Polydisperse Mixtures Chemical Engineering Science: 52(2), 177-181 (1997).

Browarzik D.; Continuous Thermodynamics of Binary Associating Systems, Fluid Phase Equilibria: 254(1-2), 174-187 (2007).

Cotterman R.L., R. Bender y J.M. Prausnitz; Phase Equilibria for Mixtures Containing Very Components. Development and Application of Continuous Thermodynamics for Chemical Process Design, Industrial Engineering Chemistry Process Design Development: 24(1), 194-203 (1985).

Dewachtere N.V., F. Santaella y G.F. Froment; Application of a Single-Event Kinetic Model in the Simulation of an Industrial Riser Reactor for the Catalytic Cracking of Vacuum Gas Oil, Chemical Engineering Science: 54, 3653-3660 (1999).

Información Tecnológica Vol. - 19 No3 - 2008 
Fang W., y Q. Lei; Continuous Thermodynamic Correlation and Calculation of Vapor Pressure and Vapor-Liquid Equilibrium Constant of Hydrocarbon Fuel Fraction, Fluid Phase Equilibria: 213(1-2), 125-138 (2003).

Félix-Flores M.G.; Caracterización de las Fracciones Pesadas del Petróleo. Tesis de Maestría, Dpto. Ing. Química, Instituto Tecnológico de Celaya, México (2001).

Félix-Flores M.G.; Simulación del Proceso FCC: Caracterización de las Corrientes de Alimentación y Productos del Riser a Través de la Función de Distribución Gamma. Tesis de Doctorado, Dpto. Ing. Química, Instituto Tecnológico de Celaya, México (2006).

Gray M.R.; Upgrading Petroleum Residues and Heavy Oils, Primera edición, 46-48, Taylor and Francis, U.S.A. (1994).

Han I. S. y C.B. Chung; Dynamic Modeling and Simulation of a Fluidized Catalytic Cracking Process. Part I: Property and Estimation and Simulation, Chemical Engineering Science: 56, 1973-1990 (2001).

Lage P.L.C.; The Quadrature Method of Moments for Continuous Thermodynamics, Computers and Chemical Engineering: 31(7), 782-799 (2007).

Mccoy B.J.; Continuous Kinetics of Cracking Reactions: Thermolysis and Pyrolysis, Chemical Engineering Science: 51(11), 2903-2908 (1996).

Riazi M. R.; Characterization and Properties of Petroleum Fractions, Primera edición, 87-93,167-170 ASTM International Standards Worldwide, U.S.A. (2005).

Sadeghbeigi, R.; Fluid Catalytic Cracking Handbook, Segunda edición, 1-22, 47-48, Gulf Professional Publishing, U.S.A. (2000).

Stewart, W.E., M. Caracotsios y J.P, Jorensen; Parameter Estimation from Multiresponse Data, AIChE J.: 38(5), 641-650 (1992).

Vakili-Nezhaad G.R., H. Modarress y G.A. Mansoori; Continuous Thermodynamics of Petroleum Fluids Fractions, Chemical Engineering and Processing: 40(5) 431-435 (2001). 